\title{
Cervical screening in Perth and Kinross since introduction of the new contract
}

\author{
G S Reid, A J Robertson, C Bissett, J Smith, N Waugh, R Halkerston
}

\begin{abstract}
Objective-To determine changes in the cervical screening service since the introduction of the new general practitioner contract on 1 April 1990.

Design-Analysis of computerised records of cervical screening both before and after introduction
\end{abstract} of the new contract.

Setting-General practices in Perth and Kinross Unit, Tayside.

Patients - A total of 30071 women aged 21-60 on 26 general practitioner partnership lists.

Main outcome measures-Percentage average of target population for cervical. screening in each practice for first three quarters on introduction of the contract.

Results-Perth and Kinross Unit completed a computerised cervical screening call programme in July 1989 , which produced an increase from $71 \%$ to $78 \%$ in the mean percentage of women aged $20-60$ who had had cervical smear tests within 5.5 years. Six months after the introduction of the new general practitioner contract the mean population coverage was increased to $85 \%$ in women aged 21-60 and only four practices had not attained the $80 \%$ upper target compared with 10 on 1 April 1990. Detailed examination of randomly selected practices immediately before the new contract was introduced showed an average artificial list inflation of $4 \cdot 3 \%$ in health board records when compared with practice records, a hysterectomy rate of $6 \cdot 2 \%$, and an additional $3 \%$ of women who were considered to be ineligible for smear testing due to putative virginity or illness or infirmity, or both. There was a considerable shift away from use of well woman clinics $(2.7 \%$ of smears in 1990 compared with $5.6 \%$ in 1988 ) for taking cervical smears, potentially threatening the long term viability of the clinics.

Conclusion - The introduction of the new contract for general practitioners has brought about a further sustained increase in population coverage for cervical screening in a small Scottish unit with a stable population, well motivated general practitioners, and a fully integrated computerised call and recall system based on the community health index. To optimise the screening service revision of the targets levels is necessary.

\section{Introduction}

The deficiencies in the cervical cancer screening programme in the United Kingdom, which were highlighted in the mid-1980s, resulted in the Department of Health and Social Security issuing a guidance circular in 1988 which was intended to improve standards. 'It had been recognised that many women had never been screened, the recall system was inadequate, and deaths were occurring which could be prevented. ${ }^{23}$ The introduction of the new contract for general practitioners in April 1990 included a major revision of the remuneration system for cervical screening in primary care. ${ }^{4}$ Before these changes general practitioners had been paid on an item of service basis for taking cervical smears from women aged 35 or older, once every five years. Smears taken from younger women qualified for payment only if the patient had had three pregnancies. Any other smears taken in the interests of clinical care were not specifically recompensed. ${ }^{5}$ To encourage improvement of population coverage the general practitioner contract sets targets of $50 \%$ and $80 \%$ linked directly to remuneration. The populations to be covered are women aged 25-64 in England and Wales and women aged 21-60 in Scotland. The only exceptions are women who have had a total hysterectomy.

These new arrangements follow the improvements which the government had already introduced in the form of a computerised call and recall programme covering all women in the related age groups. ${ }^{6}$ Perth and Kinross Unit, in common with the rest of Tayside, has had a successful screening programme for many years and had already completed a computerised call programme of all women aged $20-60$, resulting in a coverage at the end of July 1989 of $78 \%$. The system is based on the community health index, a computerised record of all patients registered with local general practitioners or who have had a contact with the NHS in Tayside, and includes details of postcodes, thus allowing geographically localised population screening programmes.

We assessed the impact of the new contract on screening coverage and considered what improvements are indicated.

\section{Patients and methods}

The Perth and Kinross Unit laboratories serve a community health index listed population of 123000 , of whom 30071 are women aged 21-60, the current target population for general practitioner remuneration for cervical screening services. The area covered is extensive, predominantly semirural, and includes the city of Perth (population 42000) and several smaller towns. The population is fairly stable, and although there are small pockets of inner city deprivation, on the whole the area does not suffer from the more extreme social problems encountered in major cities elsewhere in the United Kingdom. There are nine practices within the city of Perth and 17 serving the rural areas, nearly all of which are centred on small country towns.

All general practitioners in the unit participate in the computerised cervical screening programme. The average eligible list size per partner in Perth and Kinross is 346 -considerably smaller than the official average practice list size in the United Kingdom of 430 eligible patients per partner. We chose six practices at 
random - four from the city of Perth and the other two in country towns.

The details of the computer programme and outcome of a formal call programme of all women aged 20-60 in the unit have been described elsewhere. ${ }^{7 \times} \mathrm{At}$ an early stage in the call programme general practitioners were asked to check patient lists for accuracy, and $6.3 \%$ of patients on the lists were found to be no longer resident in the unit. It was expected, therefore, that patient listings on the computer before the introduction of the new general practitioner contract would prove reasonably accurate. The OCCURS computer program provides comprehensive on line data on each patient's cytological history, details of current and previous smear tests, the source of the smear, follow up and management information, details of related histological results, and confirmation of notification to the patient of their smear test result. We analysed data retrieved from the computer over the three financial quarters since 1 April 1990 and performed a more detailed analysis of six practices, which provides a direct comparison of general practitioner data with information held in the mainframe computer system.

\section{Results}

Table I gives the percentage coverage for cervical smear testing of the target population-that is, in women aged 21-60 without a hysterectomy-for each of the 26 general practices. On 1 April 1990, 16 of the practices had achieved the $80 \%$ target; all had reached the $50 \%$ target. By 1 July 1990 a further four practices had reached the $80 \%$ target, and as of 1 October 1990 all but four of the practices had attained the $80 \%$ target level. In all three quarters studied the vast majority of those failing to reach the top target had already attained

TABLE I-Percentage coverage for cervical smear testing in each general practice in Perth and Kinross unit, for first three quarters on introduction of contract

\begin{tabular}{lccc}
\hline Practice & 1 April & 1 July & 1 October \\
\hline A & $87 \cdot 5$ & $88 \cdot 9$ & $89 \cdot 6$ \\
B & $76 \cdot 4$ & $83 \cdot 7$ & $84 \cdot 3$ \\
C & $85 \cdot 2$ & $86 \cdot 2$ & $86 \cdot 6$ \\
D & $88 \cdot 2$ & $89 \cdot 3$ & $89 \cdot 3$ \\
E & $81 \cdot 6$ & $83 \cdot 9$ & $86 \cdot 6$ \\
F & $74 \cdot 6$ & $79 \cdot 3$ & $83 \cdot 9$ \\
G & $89 \cdot 2$ & $91 \cdot 4$ & $93 \cdot 1$ \\
H & $83 \cdot 8$ & $85 \cdot 2$ & $84 \cdot 5$ \\
I & $69 \cdot 4$ & $71 \cdot 0$ & $71 \cdot 3$ \\
J & $79 \cdot 7$ & $84 \cdot 2$ & $84 \cdot 3$ \\
K & $83 \cdot 2$ & $84 \cdot 9$ & $84 \cdot 9$ \\
L & $73 \cdot 8$ & $78 \cdot 7$ & $81 \cdot 9$ \\
M & $71 \cdot 8$ & $74 \cdot 5$ & $74 \cdot 7$ \\
N & $63 \cdot 3$ & $67 \cdot 6$ & $67 \cdot 3$ \\
O & $86 \cdot 6$ & $89 \cdot 1$ & $90 \cdot 7$ \\
P & $88 \cdot 0$ & $89 \cdot 2$ & $89 \cdot 4$ \\
Q & $90 \cdot 7$ & $92 \cdot 0$ & $92 \cdot 4$ \\
R & $85 \cdot 1$ & $86 \cdot 9$ & $87 \cdot 5$ \\
S & $68 \cdot 4$ & $74 \cdot 7$ & $79 \cdot 3$ \\
T & $79 \cdot 1$ & $81 \cdot 7$ & $82 \cdot 2$ \\
U & $83 \cdot 7$ & $85 \cdot 4$ & $85 \cdot 6$ \\
V & $81 \cdot 9$ & $82 \cdot 7$ & $83 \cdot 9$ \\
W & $77 \cdot 7$ & $80 \cdot 9$ & $83 \cdot 5$ \\
X & $80 \cdot 0$ & $84 \cdot 2$ & $84 \cdot 7$ \\
Y & $82 \cdot 9$ & $85 \cdot 2$ & $87 \cdot 2$ \\
Z & $81 \cdot 7$ & $82 \cdot 1$ & $83 \cdot 6$ \\
\hline
\end{tabular}

TABLE II - Percentage (number) of smears taken by source

\begin{tabular}{lccc}
\hline & $\begin{array}{c}\text { April and May } \\
1988 \\
\%(\mathrm{No})\end{array}$ & $\begin{array}{c}\text { April and May } \\
1989\end{array}$ & $\begin{array}{c}\text { April and May } \\
1990 \\
\%(\mathrm{No})\end{array}$ \\
\hline Source & $3 \cdot 3(62)$ & $1 \cdot 9(34)$ & $1 \cdot 2(26)$ \\
Antenatal department & $6 \cdot 1(114)$ & $5 \cdot 5(98)$ & $4 \cdot 6(98)$ \\
Gynaecology department & $8 \cdot 5(159)$ & $7 \cdot 4(133)$ & $5 \cdot 6(119)$ \\
Family planning & $1 \cdot 4(26)$ & $0 \cdot 8(15)$ & $0 \cdot 7(15)$ \\
Colposcopy & $0 \cdot 4(7)$ & $0 \cdot 9(16)$ & $0 \cdot 5(10)$ \\
Genitourinary medicine & $5 \cdot 6(105)$ & $5 \cdot 9(105)$ & $2 \cdot 7(58)$ \\
Well woman clinic & $0 \cdot 1(1)$ & $0 \cdot 2(3)$ & $-(1)$ \\
Other & $74 \cdot 6(1394)$ & $77 \cdot 5(1390)$ & $84 \cdot 6(1796)$ \\
General practice & $100(1868)$ & $100(1794)$ & $100(2123)$ \\
\hline Total & \multicolumn{3}{|}{} \\
\hline
\end{tabular}

TABLE III-Target population statistics for six random practices on 1 April 1990

\begin{tabular}{lcccc}
\hline & $\begin{array}{c}\text { Female } \\
\text { population } \\
\text { aged 21-60 }\end{array}$ & $\begin{array}{c}\text { List inflation } \\
(\text { No }(\%))\end{array}$ & $\begin{array}{c}\text { No }(\%) \text { of } \\
\text { hysterectomies }\end{array}$ & $\begin{array}{c}\text { Eligible } \\
\text { population }\end{array}$ \\
\hline Practice & 696 & $37(5 \cdot 3)$ & $44(6 \cdot 3)$ & 615 \\
$\mathrm{D}$ & 791 & Not available & $56(7 \cdot 0)$ & 735 \\
$\mathrm{~L}$ & 1301 & $72(5 \cdot 5)$ & $99(7 \cdot 6)$ & 1130 \\
$\mathrm{P}$ & 2293 & $99(4 \cdot 3)$ & $131(5 \cdot 7)$ & 2063 \\
$\mathrm{R}$ & 2730 & $64(2 \cdot 3)$ & $140(5 \cdot 1)$ & 2526 \\
$\mathrm{~T}$ & 1839 & $77(4 \cdot 2)$ & $101(5 \cdot 5)$ & 1661 \\
$\mathrm{X}$ & 9650 & $349(4 \cdot 3)$ & $571(6 \cdot 2)$ & 8730 \\
\hline Total & & & &
\end{tabular}

TABLE IV-Population coverage for cervical smear testing in six random practices over first three quarters on introduction of contract

\begin{tabular}{lcccc}
\hline & & \multicolumn{3}{c}{$\%$ Population coverage } \\
\cline { 3 - 5 } Practice & $\begin{array}{c}\text { Eligible } \\
\text { population }\end{array}$ & 1 April & 1 July & 1 October \\
\hline D & 615 & $88 \cdot 2$ & $89 \cdot 3$ & $89 \cdot 3$ \\
L & 735 & $73 \cdot 8$ & $78 \cdot 7$ & $81 \cdot 9$ \\
P & 1130 & $88 \cdot 0$ & $89 \cdot 2$ & $89 \cdot 4$ \\
R & 2063 & $85 \cdot 1$ & $86 \cdot 9$ & $87 \cdot 5$ \\
T & 2526 & $79 \cdot 1$ & $81 \cdot 7$ & $82 \cdot 2$ \\
X & 1661 & $80 \cdot 0$ & $84 \cdot 2$ & $84 \cdot 7$ \\
\hline
\end{tabular}

a population coverage of over $70 \%$. The practices that had most problems in achieving the $80 \%$ target were usually rural and usually had a smaller number of partners.

To investigate the apparent effect of the new contract on smear taking services outside general practice we examined the source of all smears arriving in our laboratory during a comparable period (April and May) in 1988, 1989, and 1990 (table II). Although the general practitioner contribution increased by $10 \%$, utilisation of other sources decreased, the most notable decrease being the well woman clinics, which halved the number of smears submitted. The absolute figures confirm that these changes were not simply the result of general practitioners taking more smears but rather show that there was a real decrease in the number of smears being taken by these other centres.

Table III shows the population statistics as on 1 April 1990 for the six general practices which were randomly selected for more detailed study. Four of these practices were in the city of Perth and two (D and L) were in small country towns. There was an average list inflation of $4 \cdot 3 \%$ (range $2 \cdot 3 \%$ to $5 \cdot 5 \%$ ) of patients assigned to these practices who were on the community health index but were either unknown to the practice or had left their lists. The list inflation for practice $\mathrm{L}$ was not available, and this practice highlights one of the problems of the new system in that it includes on its list a relatively high population of transient hotel staff, for whom it is extremely difficult to keep accurate up to date records. The six practices had a mean hysterectomy rate of $6 \cdot 2 \%$ (range $5 \cdot 1 \%$ to $7 \cdot 0 \%$ ); this is very similar to the rate of $7 \%$ in Tayside. As might be expected the hysterectomy rate in Tayside increases in the older age groups, being $0 \cdot 1 \%$ in women aged $20-30$, $2 \cdot 5 \%$ in women aged $30-40,10 \cdot 8 \%$ in women aged $40-50$, and $16 \cdot 6 \%$ in those aged $50-60$.

Table IV shows the performances of the six randomly chosen practices during the first three quarters of the new contract. On 1 April four of these practices had achieved the $80 \%$ target; the two others achieved $74 \%$ and $79 \%$ coverage respectively. By 1 July 1990 five practices had achieved $80 \%$ coverage, and on 1 October 1990 all of the study practices had achieved a coverage of over $80 \%$. Interestingly, the percentage population coverage was maintained or increased in all practices over the quarters studied.

Table $\mathrm{V}$ gives the reasons for women in the six study practices not having had a smear test in the 5.5 years preceding 1 April 1990. They are divided into three 
main groups: $(a)$ women who positively indicated that they did not wish to have a smear test even after appropriate counselling $(0 \cdot 4 \%$ to $4 \cdot 2 \%) ;(b)$ women for whom a smear was considered clinically unwarranted or impractical $(2 \cdot 3 \%$ to $3 \cdot 7 \%)$; and $(c)$ non-responders - that is, women who failed to reply to a minimum of two contacts by general practitioners and a further letter from the laboratory and, based on General Post Office information, were still resident at the stated address $(5 \cdot 1 \%$ to $19 \cdot 6 \%)$.

TABLE $\mathrm{v}-$ Reasons for women in six random practices not having had a smear test for $5 \cdot 5$ years before 1 April 1990. Figures are numbers (percentages) of women

\begin{tabular}{lcccc}
\hline & $\begin{array}{c}\text { Target } \\
\text { population }\end{array}$ & $\begin{array}{c}\text { Refused to be } \\
\text { tested }\end{array}$ & $\begin{array}{c}\text { Testing was } \\
\text { clinically } \\
\text { unwarranted }\end{array}$ & $\begin{array}{c}\text { No response } \\
\text { to invitation }\end{array}$ \\
\hline $\mathrm{D}$ & 615 & $8(1 \cdot 3)$ & $22(3 \cdot 6)$ & $42(6 \cdot 9$ \\
$\mathrm{P}$ & 1130 & $35(3 \cdot 1)$ & $42(3 \cdot 7)$ & $58(5 \cdot 1)$ \\
$\mathrm{R}$ & 2063 & $55(2 \cdot 6)$ & $52(2 \cdot 5)$ & $200(9 \cdot 7)$ \\
$\mathrm{X}$ & 1661 & $19(1 \cdot 1)$ & $51(3 \cdot 0)$ & $261(15 \cdot 7)$ \\
$\mathrm{T}$ & 2526 & $10(0 \cdot 4)$ & $83(3 \cdot 3)$ & $469(18 \cdot 6)$ \\
$\mathrm{L}$ & 735 & $31(4 \cdot 2)$ & $17(2 \cdot 3)$ & $144(19 \cdot 6)$ \\
\hline Total & 8730 & $158(2 \cdot 1)$ & $267(3 \cdot 1)$ & $1174(12 \cdot 6)$ \\
\hline
\end{tabular}

\section{Discussion}

We were ideally placed in Tayside to examine the influence of the new general practitioner contract as we had already completed our call programme and hence any increase in coverage for cervical screening was likely to result predominantly from the changes brought about by the contract. Before the introduction of the new contract $78 \%$ of the women aged $20-60$ in Perth and Kinross had already had a cervical smear test - the call programmes had increased the level from $71 \% .{ }^{8}$ Under the new system the percentage of women aged 21-60 who had had a smear test within the past $5 \cdot 5$ years had been increased to $86 \%$ on 1 October 1990. In a previous paper we introduced our concept of true population coverage - that is, the number of women screened plus women with valid exemption reasons (they had had a hysterectomy or screening was clinically unwarranted) expressed as a percentage of the corrected population. In the randomly selected practices the mean true population coverage would be $89 \%$. The introduction of the new general practitioner contract has therefore apparently heralded a definite improvement in population coverage even in our unit, which had already, to our belief, optimised its service provision.

We believe, however, that changes to the system are necessary. Tayside was responsible for the development and introduction of the community health index computerised population database, which in the population studied had a rate of address errors of about $4 \%$. This compares favourably with reports from elsewhere in the United Kingdom of family health services authorities lists with inaccuracies of up to $32 \%{ }^{9}-$ errors of this magnitude make the general practitioner's problems of identifying the target population very difficult. There would have been advantages in England following the Scottish system of a community health index shared by health authorities and family practitioner committees. Even with the index, however, problems still exist within Tayside in identifying addresses of some women in the target group-for example, students and temporary hotel workers.

Although most of our practices achieved the $80 \%$ target, some did not, though most of these achieved over $70 \%$; so with continued effort we believe they should also be able to achieve the top target level. We are concerned, however, about the rather large gap between the current target levels of $50 \%$ and $80 \%$. We would rather see a more graduated system starting at a lower target of around $30 \%$ and proceeding in $10 \%$ increments thereafter up to $100 \%$ coverage. As the remuneration system is computerised it would be comparatively simple to introduce these alterations and it would have the considerable advantage of encouraging those practices at lower levels to continue to participate in the cervical screening programme and also give further incentive to practices at the current top level to continue to try to achieve complete population coverage. Assuming that the target levels set by the government were arbitrary it should be prepared to adjust its remuneration system.

One of the principles of the new contract was to improve choice for the patient. We must assume that the move away from well woman clinics and towards general practitioners as takers of smears is through real patient choice and is not the result of unreasonable coercion. We were concerned to note in the media the suggestion that women might be removed from a practitioner's list if they did not have their smear taken within the practice. The viability of our well woman clinics is now in doubt and it would be a great pity if the wider facilities offered by these clinics were to be lost as a result of the changes to the general practitioner contract.

Although it makes sense to exclude women who have had a hysterectomy from the target population, we believe this should apply only to those with benign disease and also be extended to include women with total cervicectomies and congenital uterine absence. We are concerned about the exclusion from the target population of women with a hysterectomy who have had premalignancy or invasive cancer previously diagnosed. We accept that there is some doubt as to the optimum follow up procedures for such women, but most practitioners would agree that at least some cytological follow up should be performed over the ensuing years. ${ }^{10}$

We think that there is also a need to consider further exclusions from the target population. Our data indicate that in about $3 \%$ of the population in the official target group a cervical smear test may be inappropriate. Virgins are not considered to be at risk from cervical cancer and yet they remain in the target group. In our unit practitioners are requested to identify specifically any woman whom they consider to be clinically unsuitable for screening - this would include putative virgins, women with severe intercurrent illness, etc. Each practitioner is then sent a list of such patients on a yearly basis for review and further action if appropriate and necessary. We believe that this is a realistic and sympathetic approach to the problem, and although it accounts for only a small percentage of women, under the current remunerative system a small percentage could make the difference between attaining a $50 \%$ or an $80 \%$ target level. This problem could be circumvented if a more extensive and graduated system of remuneration was introduced.

The taking of some necessary cervical smears does not attract additional remuneration, " including those from women over 65 who have never had a smear test; women who need more frequent smear tests; and temporary patients, who comprise $5 \%$ of women in a north London practice.

Every general practitioner in Hereford and Worcester has reached a target level $-84 \%$ of practices being in the upper target band - while in Birmingham $25 \%$ of general practitioners did not achieve any target level and only $35 \%$ had reached the top target. ${ }^{12}$ This undoubtedly reflects the expected differences between prosperous shire areas and inner city practices and adds further support to our belief that the target levels must be altered. ${ }^{13}$ In conclusion, it seems that the performance related system is leading to a sustained improvement in the levels of population coverage, but 
we hope that the government will review the system and make the necessary further minor adjustments.

We thank our clinical colleagues and staff for their continuing support with the screening programme and Mrs J Finnie and Miss L Constable for typing the manuscript.

1 Department of Health and Social Security, Health Services Management. Cervical cancer screening. London: Department of Health and Social Security, 1988. ( $\mathrm{Hc}(88) 1$.)

2 Chamberlain J. Failure of the cervical cytology screening programme. BMf 1984;289:853-4.

3 Cancer of the cervix: death by incompetence [editoria]. Lancet 1985;ii:363-4.

4 Department of Health, Welsh Office, NHS General Medical Services. Statement on fees and allowances payable to general practitioners in England and Statement on fees and allowances payable to general practi
Wales from 1 A pril 1990. London: DOH, 1990:57-9.

5 Scottish Home and Health Department, NHS General Medical Services.
Statement of fees and allowances pavable to general medical practitioners in Scotland. London: HMSO, 1986:28.1-28.5.

6 Department of Health and Social Security. Cervical cancer screening. London:

DHSS, 1985. (DA 85/8.)
OCCURS Group. Computerisation of screening for cervical cancer. Health Bull (Edinb) 1988;46: 146-52.

8 Robertson AJ, Reid GS, Stoker CA, et al. How complete can cervical screening be? The outcome of a call screening programme for women aged 20-60 years in Perth and Kinross. Cytopathology 1990;1:3-12.

9 Shroff KJ Corrigan AM, Bosher M, et al Cervical screening in an inner city Shroff KJ, Corrigan AM, Bosher $M$, et al. Cervical screening in an inner cit area: response to a call system in general practice. BMF 1988;297:1317-8.

taken after hysterectomy for cervical intraepithelial neoplasia? $\mathrm{Br} f \mathrm{Obstet}$ taken after hysterectomy
Gynaecol 1990;97:58-61.

11 Chomet J, Chomet J. Cervical screening in general practice: a "new" scenario. BMJ 1990;300:1504-6.

12 Sefton C. GP target performance widens the income gap. Pulse 1990;Dec 8:41. 13 Waugh NR, Robertson AJ. Cervical screening in general practice. BMJ 1990;301:238.

(Accepted 6 fune 1991)

\title{
The Future of General Practice
}

\section{Set menus and clinical freedom}

\author{
T C O’Dowd, A D Wilson
}

This is the seventh in a series of articles commissioned in response to the General Medical Services Committee's strategy paper "Building Your Own Future"

Department of General

Practice, University of

Nottingham Medical

School, Queen's Medical

Centre, Nottingham NG7

2UH

T C O'Dowd, FRCGP, senior

lecturer

A D Wilson, MRCGP, lecturer

Correspondence to: $\mathrm{Dr}$ O'Dowd.

$B M \mathcal{F} 1991 ; 303: 450-2$
The freedom of doctors to do what they believe to be best for the patient is now backed into a corner, and the profession has by and large accepted this situation. After a decade when the status accorded to all professions has been challenged the medical profession is becoming accountable to society while being expected to serve the individual patient. The NHS has always acknowledged societal obligations, but there has been an implicit understanding that patients will get optimal treatment even if they have to wait for it. The extent to which this understanding is under pressure can be judged from the shroud waving of doctors in the media; such behaviour is more often motivated by decent compassion than by a desire for clinical freedom.

Limitations on clinical freedom serve to protect individuals from bad doctors and enable finite health service resources to be managed. The profession is now discussing whether general practitioners can still enjoy clinical freedom as accepted patterns of clinical management emerge. ${ }^{1}$ We do not know how far individual general practitioners accept, or have knowledge of, standard practice as defined by their peers. A degree of freedom is necessary to allow for individual flair and innovation as doctors working in highly regulated environments and with strict protocols have been shown to perform suboptimally. ${ }^{2.3} \mathrm{We}$ examine how far the balance has tipped and whether clinical freedom is at risk from protocols.

\section{Freedom in the profession}

Constraints on freedom in the medical profession are not new, and indeed being a member of any profession results in loss of personal liberty. ${ }^{+}$The traditional limitations on medical practice enforced by the profession are of an ethical nature and designed to protect the patient from antisocial acts, usually of a sexual or financial nature. This aspect of etiquette now seems quaint, and the civil courts are still left to decide on the quality of clinical care delivered in a particular case. Clinical freedom has resulted in a spectrum of care ranging from excellent to wholly bad, but because of the collegial, even secretive, practice of medicine the government and the public have difficulty in finding out where a hospital or doctor lies in this spectrum. If clinical standards are still mysterious to those outside the profession other aspects of care raise obvious questions. A public mesmerised by medical advances has begun to wonder why Aunty Mabel cannot have her hip replaced for two years and why one general practitioner cannot see you for a week while another can see you on the day you request.

Doctors know that clinical freedom often masks dangerous and inefficient practices and some have rejoiced in its passing. "It died accidentally," said Hampton, "crushed between the rising costs of new forms of investigation and treatment and the financial limits inevitable in an economy that cannot expand indefinitely." On making a case for drug formularies in hospital, Petrie and Scott argued that individual clinical freedom carries with it responsibility. ${ }^{6}$ When the government limited the list of drugs available on NHS prescription some doctors saw this as interference with their clinical freedom. But Hoffenberg thought that it was a weak issue on which to defend clinical freedom. ${ }^{7}$ Indeed it showed that at that time the profession poorly understoood the balance between clinical freedom and clinical responsibility. Since the limited list controversy there has been an emphasis on clinical responsibility and a willingness to strive for quality of care.

\section{Standards and contracts}

The theory of consensus management based on data from epidemiological or clinical trials or consensus conference has been widely accepted. This acceptance, however, has not been translated into practice. The reluctance of both hospital doctors and general practitioners to change their habits in the light of scientific evidence is illustrated by the underuse of proved treatments in the secondary prevention of myocardial infarction. ${ }^{9}$ That general practitioners and others in primary care are often too busy to keep up to date with the literature and to devise clinically sound protocols will rightly concern patients and those paying for health care. That practices may intellectually accept the need for protocols and indeed may have protocols but do not have the administrative or clinical staff to put them into effect will also concern those purchasing health care on patients' behalf. Purchasers will be tempted to lay down minimum criteria for care in the form of protocols for general practice. It will then be easy to monitor that the care is being given, even though it may have the mass produced characteristics of a hamburger chain. 